\title{
Application of Educational Decision based on Artificial Intelligence in Subject Construction
}

\author{
Jie Zheng ${ }^{1, a}$, Wei Jia ${ }^{2, b}$, Zhibo Li ${ }^{2, c}$, Chongzhen Duan ${ }^{2, d}$, Guoan Liu ${ }^{2, ~ e, ~}$ \\ Xing Qi ${ }^{2, f}$, Letian Wang ${ }^{2, g}$, and Weihao Cao ${ }^{2, h}$ \\ 1. School of Tarim University, Alaer, China \\ 2. Company of Gezhouba Group Transportation Investment co., LTD, Wuhan, China \\ a1920733371@qq.com, bFuma1406652770@163.com, c1661922087@qq.com, \\ d781368745@qq.com, e1599283166@qq.com, f1960903692@qq.com, g1161330797@qq.com, \\ h1369576592@qq.com
}

\begin{abstract}
Artificial intelligence decision is the most commonly used method to deal with Al conflict. The advantages brought by $\mathrm{Al}$ are efficiency, accuracy, science and coordination. Educational decision is the process of behavior design and decision carried out by educational administrative departments, institutions or individuals in order to achieve specific educational goals. Taking computer science as an example, this paper gives the application of artificial intelligence decision making in the field of education, and summarizes the ideas of educational decision making based on specific courses and the actual construction process and conclusions.
\end{abstract}

Keywords: Educational Decision; Computer Science; Artificial Intelligence; Responsible Artificial Intelligence.

\section{Introduction}

\subsection{Artificial Intelligence}

Artificial intelligence is gradually embedded in social governance with the help of various algorithms, The emergence of algorithmic governance is closely related to the advancement of digital technology, especially after entering the 21 st century, computer science has made great progress, Greatly enhance the ability to deal with information-based regulatory activities and complex coordination problems. In the context of smart cities, algorithmic decision systems should promote the intelligent management of urban resources and processes. For example, in the fields of energy, transportation, education and crime, it should provide more appropriate services to citizens. Take traffic management as an example.

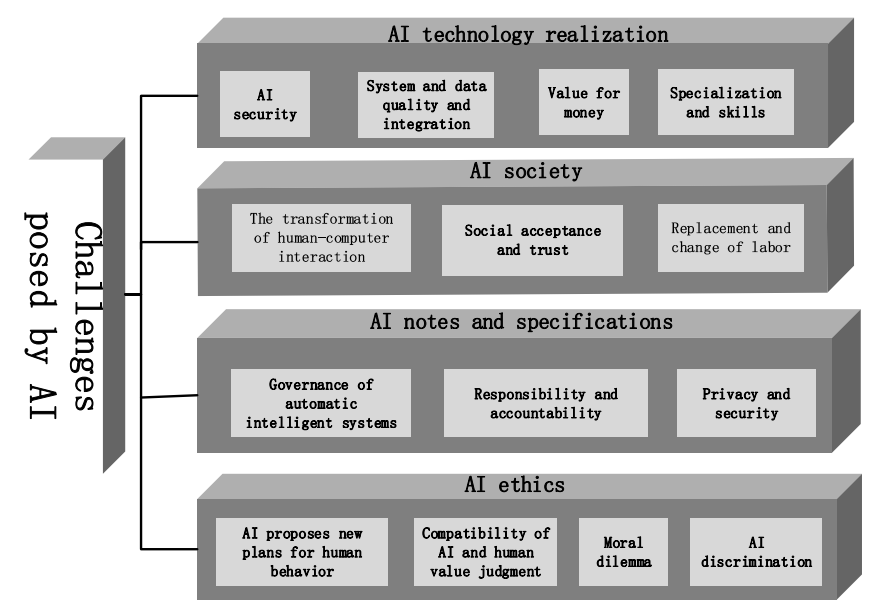

Fig 1. Four AI challenges [1] 
The challenges brought by AI include four aspects: AI society, AI technology realization, AI notes and specifications, and AI ethics. AI society includes the replacement and transformation of labor, social acceptance and the transformation of new hires and human-computer interaction. The realization of AI technology includes AI security, system and data quality, integration, cost performance, specialization and skills. AI laws and regulations include the governance of automated intelligent systems, responsibility and accountability, privacy and security. AI ethics includes AI proposes new plans for human behavior, the compatibility of AI and human value judgments, moral dilemmas and AI discrimination.

The current autonomous technology is booming. Traffic decision will be connected to the network in a separate autonomous computer. The traffic system can be achieved by setting signal lights, adjusting driving parameters, and dynamically adjusting public transportation according to actual needs. optimization.

\subsection{Educational Decision}

Educational decision is an integral part of social and cultural public policy, its essence is the process of expression and integration of different social subjects to their educational interests.[2] Good educational decisions are the precursor to good results.

\subsection{In Order to Make the Right Decision:}

Follow the general steps and methods of scientific decision. Including grasping the situation, determining the target; mass participation, democratic discussion; multiple options, comparison and optimization; thoughtful, decisive decision; experiment and demonstration, general implementation, etc.

Establish decision staff and advisory bodies. Including selecting talents, establishing institutions, clarifying tasks, and paying attention to methods.

Improve the quality of decision of education managers. The decision makers are required to have innovative spirit, scientific literacy, democratic style, and determination and courage.

The active introduction and penetration of artificial intelligence into the education field has greatly promoted the reform and innovation of educational decision, realized the transformation of education and teaching from empirical decision into current data-driven decision, and better promoted educational decision. Intelligent construction and development.[3]

\section{Meaning of AI Decision}

There are different opinions on the definition of artificial intelligence decision. Although no consensus has been reached, it can be divided into three categories.

\subsection{Ability}

AI decision is the ability to guide, manage and monitor the AI activities of an organization, which mainly includes the process of tracking, recording, and auditing activities. The definition of industrial intelligence decision emphasizes a governance capability based on model tracking and recording to achieve the transparency, credibility and compliance of artificial intelligence.[4]

\subsection{Process}

AI decision is an explainable, transparent and ethical way, a framework and process that guides the design, development and deployment of AI, mainly including action guidelines (principles) and systematic processes [5].Different from the connotation of capability, this definition emphasizes the interpretability, transparency and standardization of AI decision. 


\subsection{Method}

The mainstream in academia believes that AI decision is a way to promote AI advantages and reduce AI risks. It emphasizes the means and tools of AI decision[6]. This is also the definition of the responsibility of all mankind for AI decision, that is, through the integration of technology, standards, ethics, ethics, education and society and other factors influencing factors, finally building a trustworthy AI---Responsible_AI for mankind.[7]

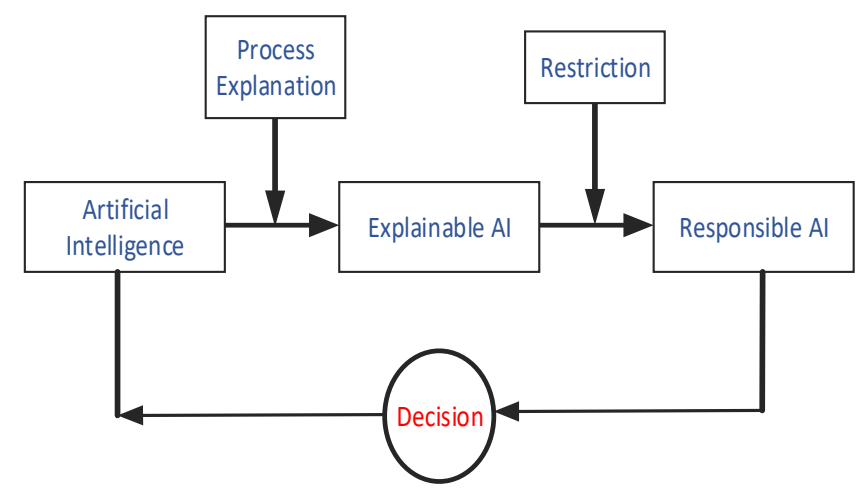

Fig 2. The connotation of AI

After explaining the process of artificial intelligence, you can explain its behavior and continue to restrict it, so that artificial intelligence has a sense of responsibility. Finally, a decision is formed and a closed loop is formed.

\section{Framework of AI Decision}

AI decision generally uses hierarchical analysis methods. The article gives a hierarchical model of AI decision. The model divides AI decision into three levels, namely, the technical layer, the restriction layer, and the application layer.[8]

\subsection{Technology}

The technical layer is the basis of AI decision, through various means to obtain the most optimized results, the main content includes: data governance, algorithm restrictions and standard setting. Data and algorithms are the main technical means of artificial intelligence at this stage, so AI decision should pay attention to data governance and algorithm responsibilities.

\subsection{Restriction}

The restriction layer is an intermediate layer that involves the ethical indicators, principles, and restrictions of AI decision. Provide a starting point and an end point for the calculation of artificial intelligence, and ensure the finiteness of artificial intelligence decision. The restriction layer is more like a virtual machine built for the operation of decision programs. It opens up memory during decision operations, records logs and releases memory after outputting the results.

\subsection{Application}

The application layer is the outer layer directly input to the artificial intelligence decision environment, and it does not contain learning processes and methods. The application layer contains a variety of parameters, sampling the real situation into specific parameters, and accessing machine learning functions. The requirements, regulations, and ethics that cannot be accurate to the computer are made into standards, combined with different target groups to make dynamic adjustments, balance the relationship between humans and machines, and weigh conflicts of interest.[9] 
Volume 15 (2021)

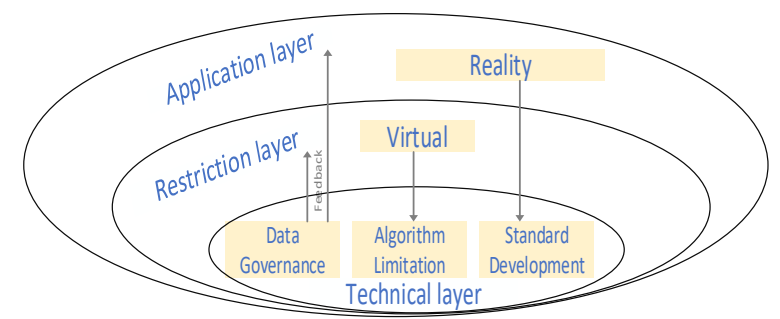

Fig 3. The connotation of artificial intelligence

The application layer is realistic, which includes a virtual restriction layer, and the technical layer contained inside includes data governance, algorithm restrictions and standard formulation.

\section{Technology of AI Decision}

\subsection{Weight Control of Decision}

In common artificial intelligence decision, it is often necessary to consider the weight between machines and ethics. The common correction method is the threshold-intervention method. That is, the application layer threshold is set in advance, and when the parameter exceeds the threshold, manual intervention is introduced.

In practical applications, the decision result closest to the objective function is selected as the decision estimate. The model is as :

$$
u_{i}= \begin{cases}1, & K_{A I}-K<0 \\ 0 & , K_{A I}-K \geq 0\end{cases}
$$

Where $u_{i}$ is the weighting factor of parameter $i, K$ is the application layer threshold. When $K_{A I}-K<0$, The model system determines that the machine decision is operating normally, and the data governance weight of the machine is $100 \%$; on the contrary, the confidence of data governance is 0 , and the model reports an error, Manual intervention is involved.

The two-stage model judges the weight between the machine and the ethics, and avoids the wrong and degraded decision result when the machine runs abnormally. But the two-stage model is a simple, direct judgment method that does not require machine learning. It can only be used as a physical process of parameter calculation, and does not have the meaning of intelligence.

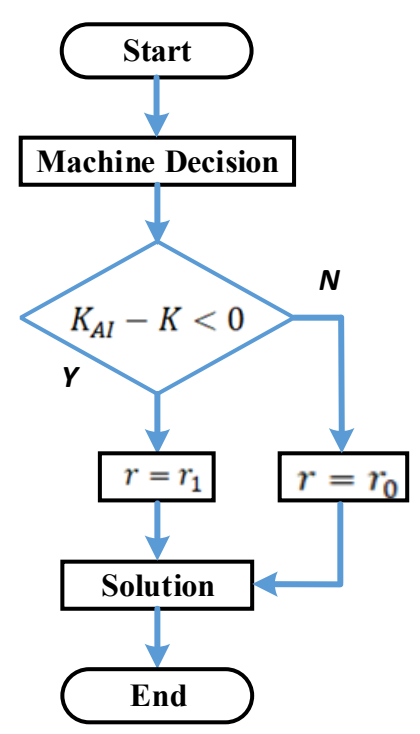




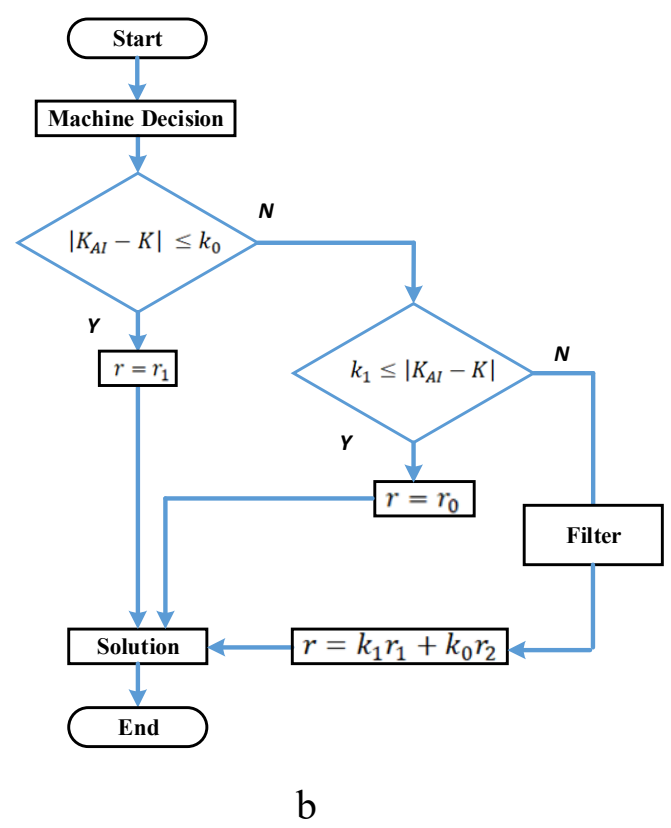

Fig 4. Comparison of decision processes

\subsection{AI Decision--Three Stage Decision}

In the actual application of artificial intelligence, decision generally will reasonably weigh the weight of machines and ethics.

If the machine does not experience major deterioration, the decision measurement result will not exceed the threshold. That is to say, the initial decision error is ignored, and the three-stage weight reduction factor model is used to set the weights for machine learning. The model is as :

$$
w_{i}=\left\{\begin{array}{ccr}
1, & \left|K_{A I}-K\right| \leq k_{0} \\
\frac{k_{1}-\left|K_{A I}-K\right|}{k_{1}-k_{0}}, & k_{0}<\left|K_{A I}-K\right|<k_{1} \\
0, & k_{1} \leq\left|K_{A I}-K\right|
\end{array}\right.
$$

Where $w_{i}$ is the weighting factor of parameter $i, k_{1} k_{0}$ is the threshold value of the two parameters set in the difference. When the difference is smaller than $k_{0}$, The weight factor of machine parameters is 1 , and the model does not involve ethical decision; When the difference is bigger than $k_{1}$, The weight factor of the machine parameters is 0 , the machine is running abnormally, and manual intervention is introduced; When the difference is between $k_{1}$ and $k_{0}$, The weight factor is used as the filter variable of machine learning to estimate the optimal weight state, and the optimal decision result obtained at real time is:

$$
r=w r^{\cdot}=k_{1} r_{1}+k_{0} r_{2}
$$

Where $r$ is the final decision result, $r$ is the fusion decision results.

Through the introduction of decision-making principles in Chapters III and IV, this chapter selects the virtual reality combination method, uses the Kalman filter method to combine the two data, and captures reasonable decision results through deep learning. It also analyzes the possibility of intelligent decision-making in the construction of educational disciplines, and gives a virtual + reality tight combination decision-making plan. 


\section{Comparison of AI Decision Results}

The experiment selected some parameter change data under strict ethical parameters and relaxed ethical parameters for decision simulation. In Fig.6,it can be seen that when the strict ethical parameters are controlled or the ethical parameters are relaxed, the artificial intelligence decision can obtain more optimized decision results, and the result output is more stable.
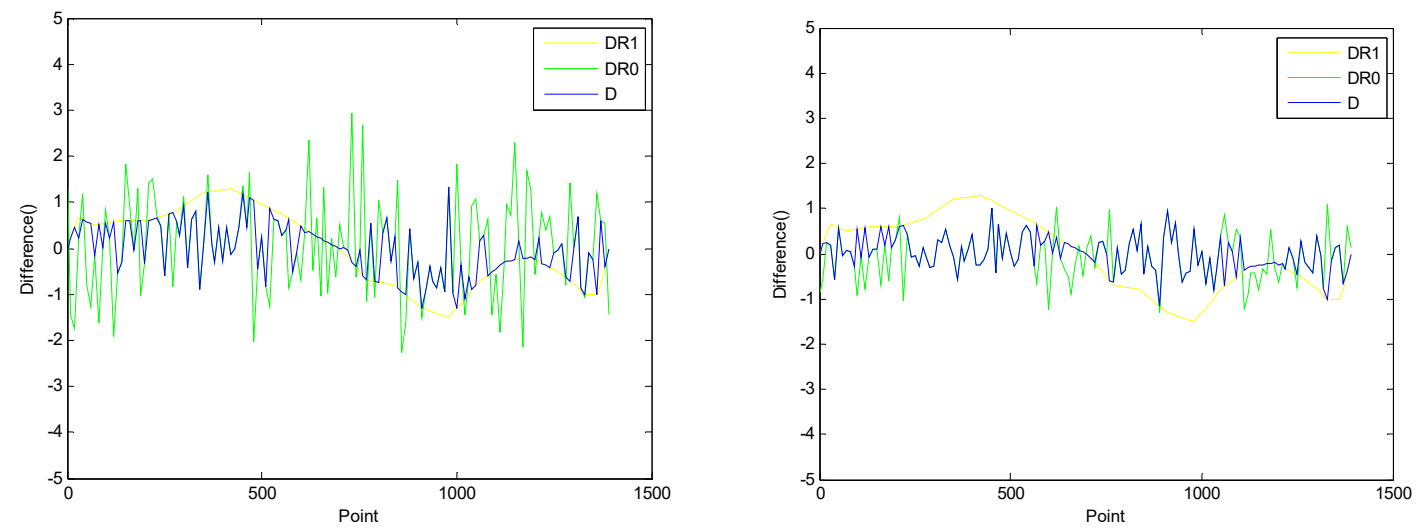

Fig 5. Two-stage decision comparison
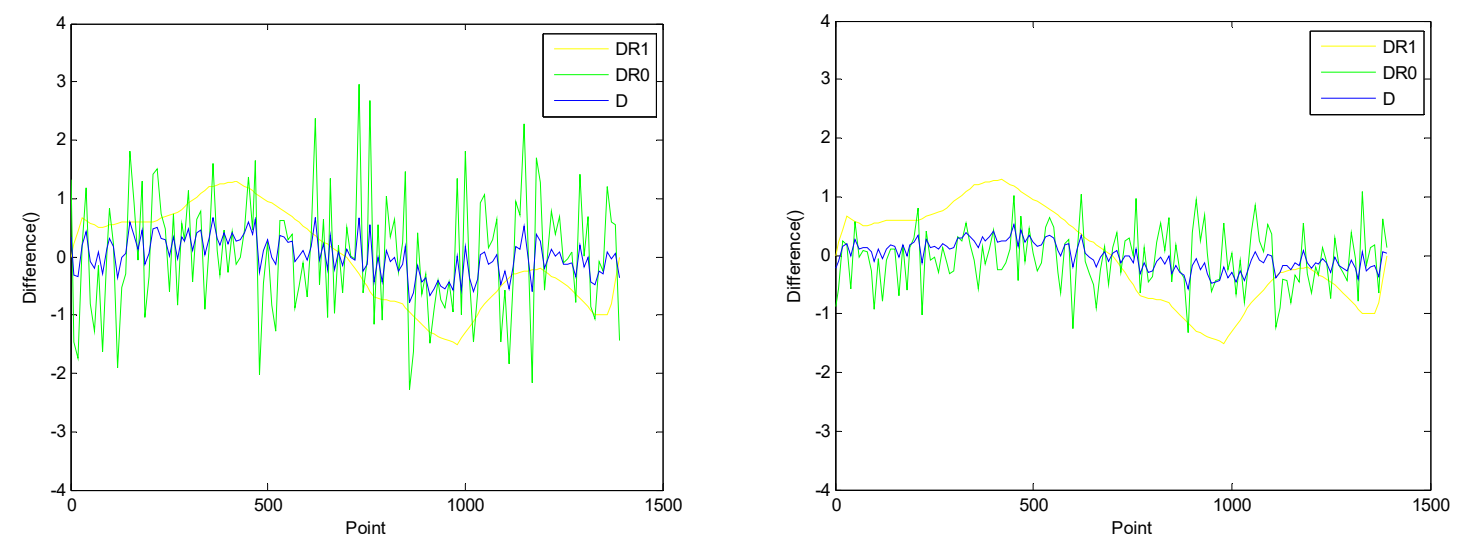

Fig 6. AI decision comparison

Comparing Fig.5 and Fig.6, it can be seen that no matter under strict ethical parameters and relaxed ethical parameters, artificial intelligence decision can obtain decision results that are more suitable for the objective function.

\section{Summary}

Scientific and standardized decision guarantees the quality of the education process and promotes the inherent requirements of scientific and democratic educational decision. The application of artificial intelligence decision in the field of educational decision can help educational decision subjects explain and analyze educational issues, formulate educational policies, and predict the future development direction of education. In the process of advancing the modernization of the education governance system and governance capabilities, make full use of technologies such as deep mining of big data and artificial intelligence decision to comprehensively collect and analyze education and teaching information, and formulate personalized education programs that meet their own characteristics and national conditions, including effective data, The powerful information will comprehensively improve the quality of education, optimize the educational decision plan, give play to the tension between disciplines, promote the science and democracy of educational decision, and greatly increase the public's recognition and support for educational decision. Therefore, the 
application of big data mining and decision technology science to educational decision can comprehensively improve the ability of educational big data speculation and analysis, effectively optimize educational decision, and fundamentally strengthen the depth and breadth of decision. Artificial intelligence decision is important for promoting major educational decision. Implementation is of great significance.

\section{References}

[1] WIRTZ B W, WEYERER J C, GEYER C. Artificial Intelligence and the Public Sector Applications and Challenges [J]. International Journal of Public Administration, 2019,42 (7): 596 - 615.

[2] Wei Lina, Shen Jinlu. Educational decision-making mechanism in the United States: subject composition, principle of action and key model [J]. World education information,2020,33(05):34-39.

[3] Qi Xin-yu. On quality discrimination and quality assurance of educational decision-making Process [J]. Shanghai Education Research,2006(2):19-22.

[4] AI governance: Ensuring your AI is transparent, compliant, and Trustworthy [EB/OL]. [2021-05-15]. https://www.ibm.com/analytics/common/smart papers/ai-governance-smartpaper / \# ai-governancedelivers.

[5] AI Governance: The path to responsible adoption of artificial intelligence [R/OL]. [2021-05-15]. https://www.asianscientist.com/wp-content/uploads / 2020/07 / AI-governance -Whitepaper-Basis - AI. PDF.

[6] ULNICANE I, KNIGHT W, LEACH T, et al. Framing Governance for a Contested Emerging Technology: Insights from AI Policy [J]. Policy and Society, 2020,40 (2): 1-20.

[7] WEARN O R, FREEMAN R, JACOBY D M. Recovery AI for conservation [J]. Nature Machine Intelligence, 2019,1 (2):72-73.

[8] GASSER U, ALMEIDA V a. layered model for AI governance [J]. IEEE Internet Computing, 2017,21 (6): 58-62.

[9] REDDYS, ALLANS, COGHLANS, et al. A governance model for the application of AI in health care [J]. Journal of the American Medical Informatics Association, 2020, 27 (3): 491-497. 